Trauma Berufskrankh 2018 20 (Suppl 4):S265-S271 https://doi.org/10.1007/s10039-018-0374-y Online publiziert: 7. Mai 2018 (c) Springer Medizin Verlag $\mathrm{GmbH}$, ein Teil von Springer Nature 2018

CrossMark

\author{
H. Riepenhof ${ }^{\prime} \cdot$ S. McAleer ${ }^{2} \cdot$ R. Delvescovo ${ }^{3} \cdot$ S. Lindenmeyer $^{4} \cdot$ F. Sikorski ${ }^{1}$ \\ 'Sportprävention, Sportrehabilitation, BG Klinikum Hamburg, Hamburg, Deutschland \\ ${ }^{2}$ British Athletics, Lee Valley Athletics Centre, London, Großbritannien \\ ${ }^{3}$ Medical and Performance Department, AS Roma Football Club, Rom, Italien \\ ${ }^{4}$ Rehaklinik Zihlschlacht, Zihlschlacht, Schweiz
}

\title{
Sportverletzungen - Möglichkeiten und Grenzen der konservativen Therapie
}

(2,7 Verletzungen pro Profisportler pro Jahr) und im Eishockey (2,7 Verletzungen pro Profisportler pro Jahr) und etwas seltener im Fußball (2,5 Verletzungen pro Profisportler pro Jahr) und im Basketball (1,9 Verletzungen pro Profisportler pro Jahr) auf [4]. Lediglich $10 \%$ der Amateursportler verletzen sich durchschnittlich 1-mal im Jahr. [4-7].

Absolut entstehen die meisten Sportverletzungen im Fußball, was trotz abnehmender Tendenz darauf zurückzuführen ist, dass noch immer $50 \%$ der aktiv Sporttreibenden Fußballer sind. Im Frauenamateursport nimmt die Anzahl aktiver Fußballerinnen seit Jahren stetig zu. Somit finden im Fußball und im konstant beliebten Handball über ein Drittel aller weiblichen sportlichen Aktivitäten statt. Die Aktivität in traditionell starken Sportarten des Frauenamateursports, wie z. B. Turnen, nimmt im Gegensatz dazu seit Jahren stetig ab [2].

Es gibt weder im Profi- noch im Amateursport zuverlässige Zahlen, wie viele der Sportverletzungen konservativ und wie viele operativ behandelt werden. Die Aussagen variieren stark, je nachdem ob in Kliniken, Praxen oder Sportvereinen retrospektiv Daten erhoben wurden. Fasst man die unterschiedlichen Angaben zusammen, so lässt sich gemittelt eine Größe von etwa 4,7\% (zwischen $1,8 \%$ und $8,3 \%)$ bestimmen. Es ist allerdings erforderlich, dies in aussagekräftigen Studien prospektiv zu erheben [3].

\section{Muskelverletzungen im Sport}

Fast ein Drittel aller Sportverletzungen betreffen den Muskel [8-10]. Und auch wenn die Muskelverletzung als eine der häufigsten Sportverletzungen primär konservativ behandelt wird, gibt es auch hier Grenzen und Indikationen zur operativen Therapie.

Verletzungen der ischiokruralen Muskulatur sind die häufigsten intrinsischen Muskelverletzungen im Sport [10], und es lässt sich anhand dieser Muskelgruppe sehr gut verdeutlichen, wann und unter welchen Bedingungen eine Indikation zur Operation nach zugezogener Verletzung besteht. Außerdem sollte den sog. Hamstrings besondere Beachtung geschenkt werden, da vermutlich aufgrund der v. a. im Profisport steigenden Trainings- und Spielintensität eine zunehmende Inzidenz über das letzte Jahrzehnt beobachtet wurde [9].

\section{Klassifizierung von intrinsischen Muskelverletzungen}

Es gibt verschiedene Möglichkeiten, intrinsische Muskelverletzungen einzuteilen. Die gängigen Verfahren sind dabei die rein radiologische Betrachtung, entsprechend der in der Magnetresonanztomographie (MRT) gefundenen Verletzungsgröße, und eine im Münchner Konsens entstandene Klassifikation $[8,11$, 12]. Die $\bullet$ Abb. 1, 2, 3 und 4 zeigen Muskelverletzungen verschiedener Schweregrade. sich deutlich häufiger verletzen als Amateursportler [4-7]. Im Profisport treten Verletzungen insbesondere im Handball 

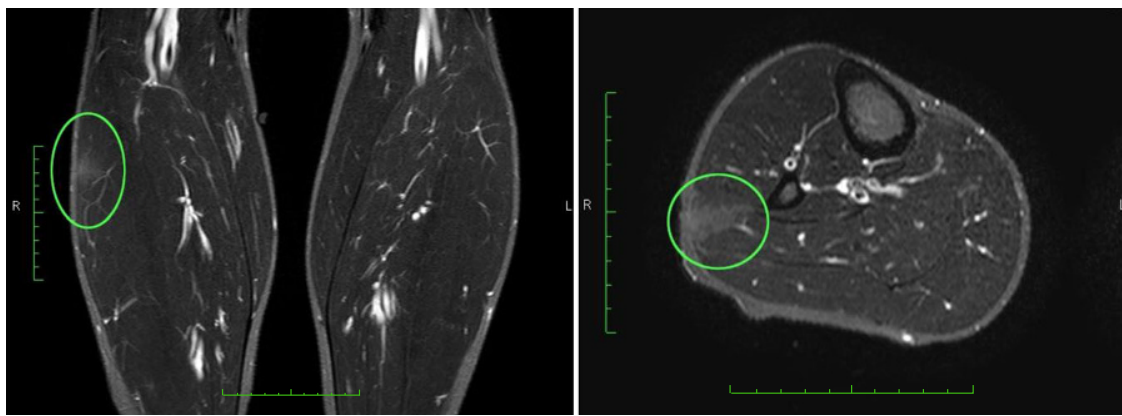

Abb. 1 ム MRT-Aufnahme einer Grad-0-Verletzung. Lediglich vorhandene Ödembildung im Muskel. Die Anordnung der Muskelfasern ist weiterhin erhalten, kein Funktionsverlust, wenig bis kein Schmerz
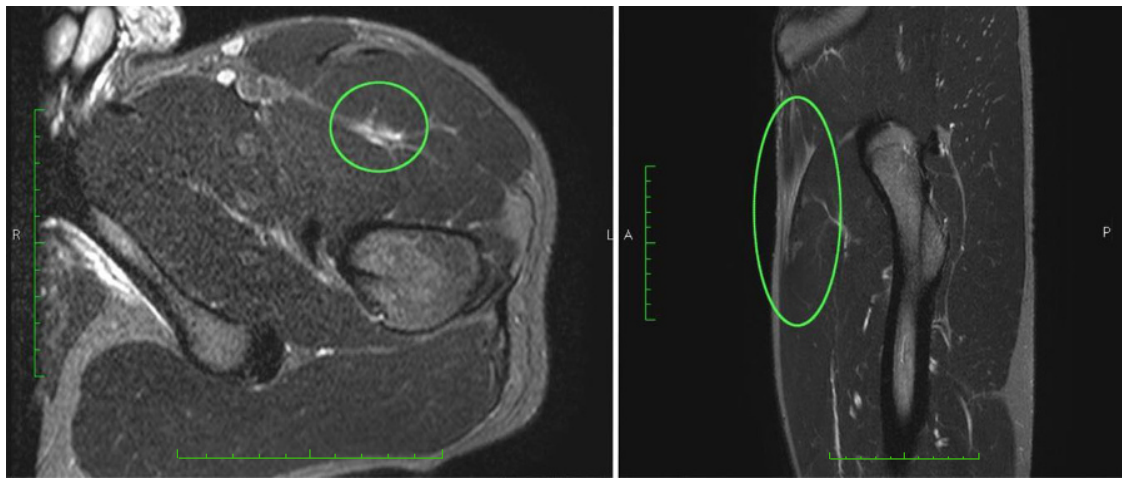

Abb. 2 ム MRT-Aufnahme einer Grad-1-Verletzung. Überdehnung, Zerrung, Zerreißung nur sehr weniger Muskelfasern, leichte Entzündungszeichen, Schwellung, bewegungsabhängiger Dehnungsschmerz

Der wesentliche Unterschied dabei ist, dass die in der MRT darzustellende Ödembildung im Muskel radiologisch die sog. Grad-0-Verletzung darstellt. Nach dem Münchner Konsens ist dies aber bereits eine Verletzung ohne strukturellen Schaden und somit eine Grad1- oder Grad-2-Verletzung. Ohne dass Muskelfasern in ihrer Struktur verletzt sind, wird hier zwischen 2 Formen der "muscle disorder" differenziert:

- die erste Form aufgrund einer „overextension“, die sich dann als "fatigue induced muscle disorder" (1A) oder "delayed onset muscle soreness", sog. DOMS (1B) darstellt;

- die zweite Form aufgrund von neuromuskulären Dysfunktionen des Muskels.

Bei beiden Formen existieren 2 Typen, wobei die einen wirbelsäulenbezogen (2A) und die anderen muskelbezogen (2B) sind. Die $\bullet$ Tab. 1 stellt die gängigsten Klassifizierungen von Muskelverletzungen in der Übersicht dar.

\section{Möglichkeiten der konservativen Therapie}

\section{Muskelverletzungen Grad 0 bis 2}

Muskelverletzungen des Grades 0 bis 2 sind die Domäne der konservativen Therapie. Lediglich große Hämatome oder drohende Kompartmentsyndrome bilden hier die Ausnahme und werden weiter unten besprochen.

In aktuellen Lehrbüchern [13-17] werden diverse Therapiemethoden aufgeführt. Viele davon sollen zur konservativen Therapie dienen. Im Folgenden sind diese aufgeführt und werden kritisch diskutiert.

Es gibt auf Grundlage weniger klinischer Studien einen großen Konsens bezüglich der Behandlungsmaßnahmen der ersten 5 Tage nach erlittener Muskelverletzung, der sog. Sofortmaßnahmen [18-21].

\section{RICE-Protokoll oder PECH-REGEL}

Die als RICE-Protokoll oder PECH-REGEL beschriebene Therapie, bestehend aus Pause („rest“), Eis („ice“), Kompression („,compression“) und Hochlagerung („elevation“), ist die am weitesten verbreitete Methode der Sofortmaßnahmen und Therapie über die ersten 3 bis 5 Tage, unabhängig von der Verletzungsschwere. [22-24].

Die Immobilisierung in der ersten Phase soll eine weitere Blutung und damit Vergrößerung des Hämatoms verhindern.

Die Anwendung der intermittierenden Kryotherapie soll zum Stoppen der Blutung und resultierend zu kleinerem Hämatom führen. Entzündungsreize sollen reduziert und dadurch die Reparatur des verletzten Muskels beschleunigt werden. Ein eindeutiger Nachweis, dass dieser Ablauf auch erfolgt, fehlt. Lediglich die gute analgetische Wirkung der Kryotherapie ist ausreichend belegt [25]. Allerdings wird eine Schädigung der kleinsten Gefäße durch intensive Kryotherapie vermutet. Aktuelle Arbeiten empfehlen daher als Kompromiss die Applikation von Kälte um die $14^{\circ} \mathrm{C}$. So soll der Lymphabfluss gefördert und die Schädigung der Gefäße verhindert werden.

Durch eine mäßige Kompression soll der intramuskuläre Blutfluss reduziert werden, was sowohl auf die Hämatomgröße als auch auf die intramuskuläre Entzündungsreaktion einen positiven Effekt haben soll. Auch eine konsequente Hochlagerung soll dies unterstützen und darüber hinaus das Ausmaß der begleitenden Weichteilschwellung mindern.

\section{Physiotherapeutische Verfahren}

Insbesondere physiotherapeutische Verfahren sind bei der Behandlung von Muskelverletzungen weit verbreitet und bilden sozusagen die Basis der Behandlungsoptionen. Zunächst wird so die in der Literatur eindeutig als vorteilhaft beschriebene frühfunktionelle Mobilisierung sichergestellt. Wesentliches Argument ist die Förderung der Revaskularisation im geschädigten Gewebe und eine schnelle Reinnervation des heilenden Muskels. Durch funktionelle Beübung wird die fibröse Narbenbildung unterdrückt, sodass viskoelastische und kontraktile Anteile des Muskelgewebes optimal regenerieren können. [26-29]. 
Ebenso konnte als Bestandteil der physiotherapeutischen Maßnahmen nachgewiesen werden, dass durch eine schmerzfreie Dehnung des heilenden Muskels (oder auch die weniger "gefährliche“ Variante der aktiven Streckung der antagonistischen Muskulatur) die Zeit zur Rückkehr zum Training durch Tonusreduktion, Durchblutungsförderung und Längengewinn des Muskels reduziert werden kann [30, 31].

Weitere die Heilung begünstigende Verfahren betreffen weniger den geschädigten Muskel selbst, sondern eher das umgebende Gewebe und insbesondere die Rumpfmuskulatur. Durch das Training der Rumpfmuskulatur (CoreTraining) kann der Heilverlauf positiv beeinflusst werden und die Gefahr von Reverletzungen nach Ausheilung der Muskelverletzung vermindert werden. Daher sollte bei jeder Verletzungsform ein additives Training der Rumpfmuskulatur erfolgen [22-24, 32, 33].

\section{Kinesiotape}

Die sog. neuromuskuläre Bandage, das Kinesiotape, ist im Profisport etabliert und gehört zu den Standardverfahren bei jeder Art von Verletzung. Im theoretischen Modell soll durch das Tape über die Faszie des Muskels die Muskelspannung reduziert werden und dadurch therapeutisch und analgetisch wirken. Allerdings bedarf es weiterer wissenschaftlicher Arbeiten, um diesen funktionellen Vorteil zubelegen. Nicht zuletzt aufgrund der großen Farbvarianz ist aber von einem ausgeprägten positiven Effekt auf die Psyche des Sportlers auszugehen, der die großzügige Anwendung rechtfertigen kann.

\section{Orale Therapeutika}

Als orale Therapeutika werden Bromelain, Aescin oder Ähnliche empfohlen. Hierdurch soll eine partielle Fibrinolyse ausgelöst werden, die dann antientzündlich wirkt. Auch hier ist die Gabe im Sport weit verbreitet und wahrscheinlich auch aufgrund einer gewissen $\mathrm{Ne}$ benwirkungsarmut akzeptiert. Dennoch gibt es bisher keinen angemessenen wissenschaftlichen Nachweis, der die Wirksamkeit und Vorteile einer derartigen Behandlung belegt [23].

Trauma Berufskrankh 2018 20 (Suppl 4):S265-S271

https://doi.org/10.1007/s10039-018-0374-y

(c) Springer Medizin Verlag GmbH, ein Teil von Springer Nature 2018

\section{H. Riepenhof · S. McAleer · R. Delvescovo · S. Lindenmeyer · F. Sikorski}

\section{Sportverletzungen - Möglichkeiten und Grenzen der konservativen Therapie}

\section{Zusammenfassung}

Die Deutschen sind im europäischen Vergleich überdurchschnittlich aktiv und verletzen sich dabei ca. 2.000.000-mal jährlich, wobei Profisportler sich deutlich häufiger verletzen als Amateursportler. Die häufigsten Verletzungen im Sport sind Muskelverletzungen insbesondere der ischiokruralen Muskulatur. Die Behandlung dieser Verletzungen ist die Domäne der konservativen Therapie, wobei verschiedenste Verfahren Anwendung finden. Die gängigsten Verfahren werden vorgestellt und hinsichtlich ihrer Evidenz analysiert. Trotz der großzügigen Nutzung dieser zur konservativen Therapie empfohlenen Maßnahmen insbesondere im Profisport sind nicht alle Verfahren evidenzbasiert und ggf. mit erheblichen Risiken assoziiert. Darüber hinaus gibt es Grenzen der konservativen Therapie, die auch bei Muskelverletzungen einen operativen Eingriff rechtfertigen. Abgesehen von drohenden Kompartmentsyndromen, die ausschließlich operativ zu behandeln sind, sollte bei anderen Muskelverletzungen die Indikation zur Operation eher zurückhaltend und als Einzelfallentscheidung gestellt werden. Denn auch zunächst konservative Therapieversuche zeigen nach verzögerter Versorgung sehr gute Ergebnisse, und insbesondere diverse Beispiele aus dem Profisport auf Champions-League-Niveau belegen, dass auch drittgradige, ansatznahe Abrisse der Hamstrings ein Return-toCompetition ermöglichen.

Schlüsselwörter

Muskelverletzungen · Hamstrings .

Ischiokrurale Muskulatur $\cdot$ Rehabilitation . Operation

\section{Sports injuries—possibilities and limits of conservative treatment}

\section{Abstract}

Germans have an above average participation in sports in a European comparison and sustain approximately 2 million sports-related injuries per year. Professional athletes sustain injuries much more often than amateur athletes. The most common injuries in sports are muscular injuries, especially injuries of the ischiocrural muscles. Treatment of these injuries is the domain of conservative treatment using a variety of methods. The most common treatment modalities are presented and analyzed with respect to their evidence base. Despite extensive use of the measures recommended for conservative management, especially in professional sports, not all treatment modalities are evidence-based and potentially associated with significant risks. In addition, there are limits to conservative treatment, which justify surgical treatment of muscle injuries. Apart from impending compartment syndromes, which must be treated only by surgery, the indications for surgery in muscle injuries should be reserved for difficult cases and after careful consideration. Even initial attempts at conservative treatment have demonstrated good results after protracted care. Particular examples exist within professional soccer at the Champions League level, demonstrating that conservative management of grade III ruptures of the hamstring close to the attachment point enables a return to competition.

\section{Keywords}

Muscle injuries · Hamstrings · Ischiocrural muscles $\cdot$ Rehabilitation · Surgery

\section{Topische Verfahren}

Topische Verfahren wie Salbenverbände mit Arnika oder Spurenelementen und Mineralstoffen, sind bei Sportlern sehr beliebt. Sie sollen der lokalen, entzündungshemmenden und schmerzlindernden Therapie dienen. Über das subjektive angenehme Empfinden hinaus konnte bisher allerdings kein eindeutiger Beleg eines positiven Effektes auf die Heilung von Muskelverletzungen gefunden werden $[26,27]$.

\section{Elektrotherapie, Ultraschall und andere thermische Verfahren}

Elektrotherapie, Ultraschall und andere thermische Verfahren der physikalischen Therapie zeigen überwiegend eine gute 

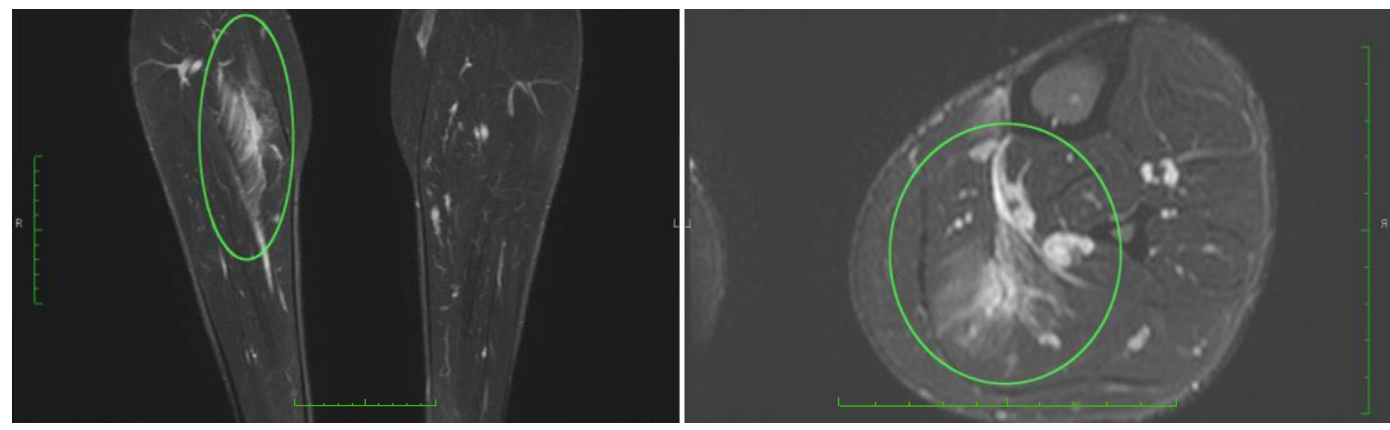

Abb. $3<$ MRT-Aufnahme einer Grad-2-Verletzung. Teilruptur, Muskelfaserriss mit schneidendem Schmerz, erheblich eingeschränkte Kraft und Bewegungsfähigkeit (ggf. tastbare Gewebslücke)
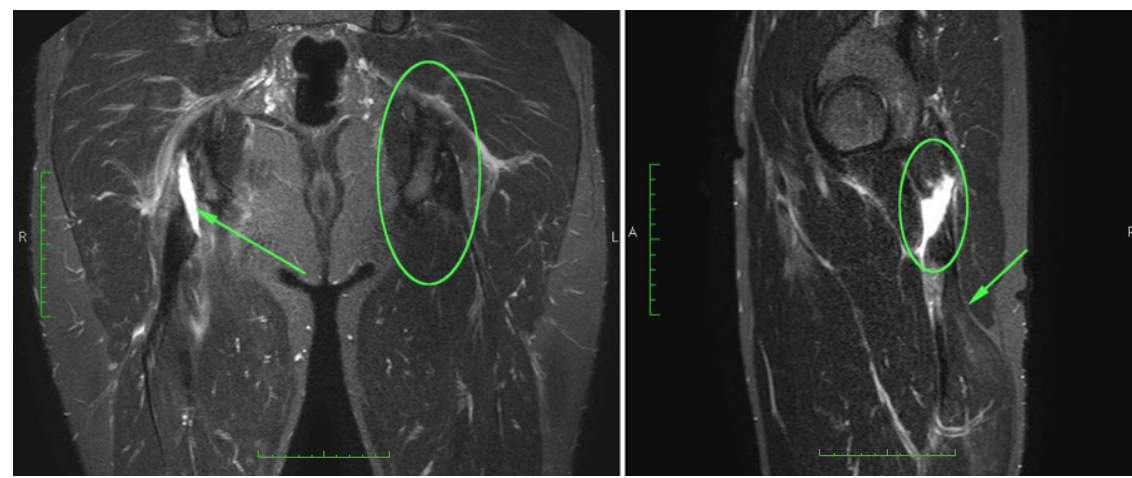

Abb. 4 ム MRT-Aufnahme einer Grad-3-Verletzung. Riss oder Abriss mit vollständigem Verlust der Funktion, tastbare, ggf. sichtbare Muskeldelle, Hämatomverfärbung durch Einblutung. Der Pfeil markiert die zurückgezogene Hamstring-Sehne, bei abgerissenem Ansatz

analgetische Wirksamkeit. Eine Förderung der Muskelfunktionalisierung durch Elektrotherapie in Kombination mit Muskelkontraktionen konnte bisher allerdings eher in Erfahrungsberichten als in streng evidenzbasierten Arbeiten nachgewiesen werden.

Auch die Ultraschalltherapie zur lokalen Durchblutungsförderung durch Applikation von Tiefenwärme insbesondere in Kombination mit Muskeldehnungen soll ebenso wie die Hyperthermie den Heilungsprozess beschleunigen. Dieses ist besonders im südeuropäischen Raum sehr beliebt. Die Anwendung von Diathermie, die ebenso durch Tiefenwärmenapplikation die Durchblutung fördern soll, bedarf aber weiterer klinischer Studien zur Sicherung eines Nachweises einer eindeutigen Wirksamkeit.

\section{Fokussierte Stoßwellentherapie}

Die fokussierte Stoßwellentherapie ist vermutlich aufgrund der Schmerzhaftigkeit der Anwendung noch kein Standardverfahren bei der Behandlung von Muskelverletzungen. Dennoch konnte am Tiermodell nachgewiesen werden, dass die ESWT (extrakorporale Stoß- wellentherapie) die Regeneration von verletzten Skelettmuskeln stimuliert und damit den Regenerationsprozess beschleunigen kann [34]. In Anwendungsuntersuchungen beim Menschen wurde bisher lediglich ein Vorteil bei der Behandlung von „Muskelverletzungen ohne strukturellen Schaden", also Grad 0-Verletzungen, beschrieben. Insbesondere die sog. DOMS ließen sich durch die Anwendung von EKSW positiv beeinflussen [35].

\section{Infiltrationsbehandlungen}

Zur Behandlung von Muskelverletzungen stehen diverse Infiltrationsbehandlungen zur Verfügung. Ziel der meisten Therapeutika soll die Beschleunigung der Heilung sein. Allerdings kann produktübergreifend festgestellt werden, dass es hier keine eindeutigen Beweise gibt.

Neben der Gabe von „heilungsfördernden Substanzen“" gehört die Injektion von Procain oder anderen Lokalanästhetika, ggf. in Kombination mit einem Steroid, zu den früher sehr verbreiteten Methoden. Auch wenn diese heute nur noch vereinzelt Anwendung finden, so sollen theoretisch 2 Aspekte der Wirkstoffkombination Vorteile erbringen:

- Einerseits soll der analgetische Effekt des Lokalanästhetikums genutzt werden, um dem Sportler ggf. zu ermöglichen, die sportliche Betätigung trotz der Verletzung fortzusetzen [36].

- Andererseits soll die antiinflammatorische Wirkung des Kortisons eine sekundäre Schädigung durch den Entzündungsprozess sowie eine Verzögerung der Heilung unterdrücken.

$\mathrm{Zu}$ Recht sind diese Verfahren inzwischen selbst im Profisport nicht mehr akzeptiert, da Sportler mit akuter Verletzung bei der Leistungsdichte, die heutzutage im Profisport vorherrscht, ohnehin keine Bestleistungen erbringen können. Die Wirkung des Kortisons, insbesondere bei Mehrfachgaben, ist besonders seit vielen medienwirksamen Pitfalls unter Profisportlern sehr umstritten.

Ausnahme ist vermutlich die Kombination von verschiedenen Mischprodukten wie Traumeel $^{\circledR}$ (Biologische Heilmittel Heel GmbH, Baden-Baden, Deutschland) in Kombinationen mit deproteinisiertem Haemoderivat aus Kälberblut (Actovegin ${ }^{\circledR}$ [Takeda Austria $\mathrm{GmbH}$, Linz, Österreich]), was besonders im Profisport propagiert wird. Zusammenfassend konnte bisher lediglich im Tiermodel ein positiver Effekt auf die Muskelzellproliferation [37] und im humanen Muskel eine vermehrte Expression von Mitochondrien in den Muskelzellen nachgewiesen werden [38]. Auch wenn eine intramuskuläre Gabe dieser Substanz derzeit nicht zu den durch die Welt-Anti-Doping-Agentur verbotenen Methoden zählt, so ist die intravenöse Gabe von deproteinisierten Haemode- 


\begin{tabular}{|c|c|c|c|c|}
\hline $\begin{array}{l}\text { Radiologische } \\
\text { Einteilung }\end{array}$ & Radiologischer Aspekt & & Klinische Darbietung & $\begin{array}{l}\text { Einteilung } \\
\text { nach Münch- } \\
\text { ner Konsens }\end{array}$ \\
\hline \multirow[t]{4}{*}{ Grad 0} & \multirow{4}{*}{$\begin{array}{l}\text { Ödem mit möglichen } \\
\text { Muskelfaserverletzungen }<5 \mathrm{~mm}\end{array}$} & \multirow{4}{*}{$\begin{array}{l}\text { Funktioneller } \\
\text { Schaden }\end{array}$} & Müdigkeitinduzierte Muskelzerstörung & Typ $1 \mathrm{~A}$ \\
\hline & & & Verzögert eintretender Muskelschmerz (DOMS) & Typ 1B \\
\hline & & & Wirbelsäulenbedingte neuromuskuläre Muskelstörung & Typ 2A \\
\hline & & & Muskelbedingte neuromuskuläre Muskelstörung & Typ 2B \\
\hline Grad 1 & $\begin{array}{l}\text { Kleinste Muskelfaserverletzun- } \\
\text { gen }<10 \mathrm{~mm}\end{array}$ & \multirow[t]{4}{*}{$\begin{array}{l}\text { Struktureller } \\
\text { Schaden }\end{array}$} & Kleine Teilruptur des Muskels & Typ 3A \\
\hline Grad 2 & Deutlicher Muskeldefekt $>10 \mathrm{~mm}$ & & Mäßige Teilruptur des Muskels & Typ 3B \\
\hline \multirow[t]{2}{*}{ Grad 3} & \multirow{2}{*}{$\begin{array}{l}\text { Kompletter Abriss des Muskels, ggf. mit } \\
\text { Retraktion }\end{array}$} & & Subtotale oder totale Muskelruptur & \multirow[t]{2}{*}{ Typ 4} \\
\hline & & & Muskelsehnenabriss & \\
\hline
\end{tabular}

rivaten aus Kälberblut weiterhin nicht erlaubt. Darüber hinaus ist die Nutzung dieser Kombinationen ein reiner Off-Label-Gebrauch, und die Erfahrungen aus der sportmedizinischen Sprechstunde zeigen, dass auch Komplikationen wie Zysten- oder Pseudozystenbildungen im Muskelgewebe mit intramuskulären Infiltrationsbehandlungen von Off-LabelProdukten assoziiert sein können. Demnach sind die Autoren der Auffassung, dass insbesondere im Amateursport auf eine solche intramuskuläre Gabe konsequent verzichtet werden sollte.

Ein weiteres, insbesondere in den USA und Europa weitverbreitetes Injektionsverfahren ist die Gabe von thrombozytenreichem Plasma (PRP), auch als plättchenreiches Plasma oder „Wachstumsfaktor" bekannt. Auch hier fehlen bisher eindeutige Belege, dass die Regenerationszeit von verletztem Muskelgewebe dadurch verkürzt wird. Des Weiteren fehlt ein Nachweis dafür, dass der verletzte Muskel nach PRP-Behandlung und Heilung weniger anfällig für Reverletzungen ist.

\section{Grenzen der konservativen Therapie}

Die chirurgische Behandlung von Muskelverletzungen ist die Ausnahme und nur bei wenigen Diagnosen indiziert.

\section{Kompartmentsyndrom}

Klare Indikation zur Operation besteht immer bei drohendem Kompartmentsyndrom. Unabhängig von der Muskelverletzungslokalisation und -klassifikati- on kann ein Kompartmentsyndrom bei ausgeprägter oder anhaltender Blutung entstehen. Selbst kleinere Verletzungen können z. B. aufgrund von medikamentöser Beeinflussung der Thrombozytenaggregation zu dieser gefährlichen Komplikation führen. Einzelne Case-Reports beschrieben dies selbst ohne aufgetretenes Trauma [39]. Daher sollte selbst bei vermeintlich weniger schweren Verletzungen stets ein Kompartmentsyndrom zum Verletzungszeitpunkt und auch im weiteren Verlauf, einige Stunden nach stattgehabtem Trauma, ausgeschlossen werden.

\section{Muskelverletzungen Grad 2 bis 3}

Die in der Literatur beschriebenen Indikationen zur operativen Behandlung von Muskelverletzungen sollten konsequent als Einzelfallentscheidung betrachtet werden.

Lediglich bei jungen Sportlern mit Abrissen der Hamstringsehnen besteht dahingehend ein Konsens, dass eine Refixation indiziert ist. Durch das operative Vorgehen soll die Leistungsfähigkeit am ehesten erhalten bleiben und das rezidivierende Auftreten von Verletzungen der gleichen Muskelgruppe verhindert werden.

Auch wenn vereinzelte vergleichende Analysen zur Patientenzufriedenheit nach operativer Versorgung von Hamstringabrissen ergaben, dass unabhängig vom Zeitpunkt des operativen Eingriffes die Zufriedenheit und Returnto-Play-Wahrscheinlichkeit mit 96,4\% gleich hoch blieb und lediglich der Zeitraum der Rehabilitationsbehandlung sich durch eine verzögerte Versorgung verlängere [40], so zeigen Metaanalysen $\mathrm{zu}$ dieser Pathologie, dass zunächst abwartendes Verhalten gut zu rechtfertigen ist. Bei der Untersuchung von 13 Arbeiten konnten sowohl bei der akuten ( $<4$ Wochen) als auch bei der verzögerten ( $>4$ Wochen) Reparatur nur minimale Differenzen zwischen Return-to-Sport, Patientenzufriedenheit, Hamstringstärke und Schmerz festgestellt werden. Darüber hinaus konnte gezeigt werden, dass die Art der operativen Versorgung, ob als primärer Repair mit Fadenankern oder als Achilles-Allograft-Rekonstruktion, vergleichbare Ergebnisse liefert [41].

Weitere Indikationen bestehen bei in der Kontinuität unterbrochenen Muskeln. Sollte eine große Distanz zwischen den verletzten Muskelanteilen vorliegen und mehr als $50 \%$ des Muskelquerschnitts betroffen sein, so wird auch hier im chirurgischen Konsens und nicht aufgrund von vergleichenden Untersuchungen eine Operation empfohlen.

Einige Beispiele aus dem Profisport zeigen allerdings auch, dass die Behandlung von drittgradigen Muskelverletzungen der Hamstrings durchaus konservativ verlaufen kann. Neben dem operativen Eingriff und den damit verbundenen Risiken stellt hier sicher auch die aufwendige Rehabilitationsbehandlung eine große Herausforderung dar und kann ein möglicher Grund sein, warum Sportler sich gegen einen operativen Eingriff bei drittgradigen Muskelverletzungen entscheiden. Trotz der Muskellücke und Heilung unter Narbenbildung scheint dies insbesondere bei 
älteren Sportlern ein Return-to-Competition nicht auszuschließen. $\mathrm{Ob}$ es nach Rückkehr zum Sport zu einer verminderten Leistungsfähigkeit kommt oder in der Folge vermehrt Verletzungen an der betroffenen Muskelgruppe oder am kompensierenden Gewebe auftreten, ist bisher noch nicht ausreichend untersucht und sollte Gegenstand weiterer wissenschaftlicher Arbeiten sein.

Auch bei weniger ausgeprägten, zum Teil nur zweitgradigen Muskelverletzungen mit großem intramuskulärem Hämatom kann in Ausnahmefällen die Indikation zur Operation bestehen. Sollten mehrfache Aspirationsversuche $\mathrm{zu}$ verschiedenen Zeitpunkten gescheitert sein, kann sich die Indikation zur Hämatomausräumung und $\mathrm{zu}$ sorgfältiger Blutstillung ergeben. Andernfalls können sich diese ausgeprägten Blutergüsse organisieren und sowohl den Muskelstoffwechsel als auch die Muskelkontraktion durch Narbenbildungen stören.

Eine weitere Grenze der konservativen Therapie von Muskelverletzungen besteht ggf. bei chronischen, also immer wiederkehrenden Muskelverletzungen. Auch wenn diese selten zu fibrösen Narbenbildungen führen, die den kontraktilen Apparat so beeinflussen, dass rezidivierend, schon bei geringer Belastung intrinsische Verletzungen auftreten, so können sie doch als Ausnahme eine operative Behandlung erfordern. Die nur einem Operateur mit viel Erfahrung bei der Therapie von Weichteilschäden vorbehaltenen Eingriffe können Narben und Adhäsionen lösen und so eine Beschwerdeminderung bewirken.

\section{Fazit für die Praxis}

- Muskelverletzungen sollten bis auf wenige Ausnahmen konservativ behandelt werden. Ausnahmen sind insbesondere drohende oder manifeste Kompartmentsyndrome.

- Auch bei höhergradigen Verletzungen zeigt die Praxis, dass ein Return-to-Competition nach nichtoperativem Vorgehen selbst im Hochleistungssport möglich ist.

- Darüber hinaus konnte in Metaanalysen dargestellt werden, dass eine zunächst abwartende Haltung und verzögerte operative Versorgung von höhergradigen Muskelverletzungen zu vergleichbaren Ergebnissen führt. Diese Erkenntnisse können durchaus einen primär konservativen Therapieversuch rechtfertigen.

- Zur konservativen Behandlung der überwiegend intrinsischen Verletzungen steht eine Vielzahl an Behandlungsmethoden zur Verfügung. Gerade im Profisport werden diese vermehrt eingesetzt, obwohl wissenschaftliche Belege für einen Behandlungsvorteil durch den Einsatz dieser Therapien bisher nicht erbracht werden konnten.

\section{Korrespondenzadresse}

\section{Dr. H. Riepenhof}

Sportprävention, Sportrehabilitation, BG Klinikum Hamburg

Bergedorfer Str. 10, 21033 Hamburg,

Deutschland

h.riepenhof@bgk-hamburg.de

\section{Einhaltung ethischer Richtlinien}

Interessenkonflikt. H. Riepenhof, S. McAleer, R. Delvescovo, S. Lindenmeyer und F. Sikorski geben an, dass kein Interessenkonflikt besteht.

Dieser Beitrag beinhaltet keine von den Autoren durchgeführten Studien an Menschen oder Tieren. Alle Patienten, die über Bildmaterial oder anderweitige Angaben innerhalb des Manuskripts zu identifizieren sind, haben hierzu ihre schriftliche Einwilligung gegeben. Im Falle von nicht mündigen Patienten liegt die Einwilligung eines Erziehungsberechtigten oder des gesetzlich bestellten Betreuers vor.

The supplement containing this article is not sponsored by industry.

\section{Literatur}

1. European Commission (2013) Special Eurobarometer 412 „Sport and physical activity“ Fieldwork: November-December. Publication 2014:8-11

2. Henke T, Luig P, Schulz D (2014) Sportunfälle im Vereinssport in Deutschland. Aspekte der Epidemiologie und Prävention. Bundesgesundheitsbla 57(6):628-637.https://doi.org/10.1007/s00103014-1964-x

3. Statistisches Bundesamt, 10 Gesundheit und soziale Sicherung, Auszug aus dem Datenreport 2016.10.3 Gesundheitliche Ungleichheit

4. VBG Sportreport 2017

5. Deutscher Olympischer Sportbund (2012) DOSB Bestandserhebung 2012, Fassung vom 15. Nov. 2012

6. TNS Opinion \& Social (2010) Eurobarometer 72.3 - Sport und körperliche Betätigung http://ec.europa. eu/public opinion/ archives/ebs/ebs_334_de.pdf. Zugegriffen: 11. Nov 2013, S93-102

7. Heinemann K, Schubert M (1994) Der Sportverein. Schriftenreihe des Bundesinstituts für Sportwissenschaft Bd. 80. Verlag Karl Hofmann, Schorndorf

8. Mueller-Wohlfahrt HW et al (2013) Terminology and classification of muscle injuries in sport: The Munich consensus statement. Br J Sports Med 47(6):342-350

9. Ekstrand J et al (2016) Hamstring injuries have increased by $4 \%$ annually in men's professional football, since 2001:A 13-yearlongitudinal analysis of the UEFA Elite Club injury study. Br J Sports Med. 2016 Jun;50(12):731-737. https://doi.org/ 10.1136/bjsports-2015-095359

10. Ekstrand J, Hägglund M, Walden M (2011) Epidemiology of Muscle Injuries in Professional Football (Soccer). Am J Sports Med 39(6):1226-1232

11. Balius R (2005) Patologia muscular en el deporte. Masson, Barcelona

12. Balius R, Rius M, Combalia A (2005) Ecografia muscular de la extremi- dad inferior. Masson, Barcelona

13. Imhoff A etal (2014) Checkliste Orthopädie, 3. Aufl. Thieme, Stuttgart

14. Rüther Wetal (2013) Orthopädie und Unfallchirurgie, 20. Aufl. Urban \& Fischer, München

15. Dickhuth HH et al (2010) Sportmedizin für Ärzte, 2. Aufl. Deutscher Ärzteverlag, Köln

16. Wülker N et al (2015) Taschenlehrbuch Orthopädie und Unfallchirurgie, 3. Aufl. Thieme, Stuttgart

17. Raschka C et al (2016) Praktische Sportmedizin. Thieme, Stuttgart

18. Mishra A, Woodall J, Vieira A (2009) Treatment of tendon and muscle using platelet-rich plasma. Clin. Sports Med 28:113-125

19. Orchard JW, Best TM, Mueller-Wohlfahrt HW, Hunter G, Hamilton BH, Webborn N, et al (2008) The early management of muscle strains in the elite athlete: best practice in a world with a limited evidence basis. Br JSports Med 42:158-159

20. Orchard JW, Best TM (2002) The management of muscle strain injuries: an early return versus the risk of recurrence. Clin J Sport Med 12:3-5

21. Quintero A, Wright V, Fu F, Huard J (2009) Stem Cells for the treatment of skeletal muscle injury. Clin. Sports Med 28:1-11

22. Hurme T, Kalimo H, Lehto M et al (1991) Healing of skeletal muscle injury: an ultrastructural and immunohistochemical study. Med Sci Sports Exerc 23:801-810

23. Järvinen $T A$, Järvinen $T L$, Kääriäinen $M$, Aärimaa $V$, Vaittinen S, Kalimo H et al (2007) Muscle injuries: optimizing recovery. Best Pract Res Clin Rheumatol 21:317-331

24. Lehto MU, Jarvinen MJ (1991) Muscle injuries, their healing process and treatment. Ann Chir Gynaecol 80:102-108

25. Hubbard TJ, Deneger CR et al (2004) Does Cryotherapy Improve Outcomes With Soft Tissue Injury? J Athl Train 39(3):278-279(Sep)

26. Chargé SB, Rudnicki MA (2004) Cellular and molecular regulation of muscle regeneration. Physiol Rev 84:209-238

27. Huard J, Li Y, Fu FH (2002) Muscle injuries and repair: current trends in research. JBone Jt Surg Am 84-A:822-832

28. Nagasaka M, Kohzuki M, Fujii T et al (2006) Effect of low-voltage electrical stimulation on angiogenic grwth factors in ischemic rat skeletal muscle. Clin ExpPharmacol Physiol 33:623-627 
29. Orchard J, Best TM, Verrall GM (2005) Return to play following muscle strains. Clin J Sport Med 15:436-441

30. Dadebo B, White J, George KP (2004) A survey of flexibility training protocols and hamstring strains in professional football clubs in England. Br JSports Med 38:388-394

31. Pas HIMFL, Reurink G, Tol JL et al (2015) Br J Sports Med 49:1197-1205

32. Sherry MA, Best TM (2004) A comparison of 2 rehabilitation programs in the treatment of acute hamstring strains. J Orthop Sports Phys Ther 34:116-125

33. Silder A, Sherry MA, Sanfilippo J et al (2013) Clinical and morphological changes following 2 rehabilitation programs for acute hamstring strain injuries: a randomized clinical trial. J Orthop Sports Phys Ther 43:284-299

34. Zissler A, Steinbacher P, Zimmermann R et al (2017) Extracorporeal Shock Wave Therapy AcceleratesRegeneration After Acute Skeletal Muscle Injury. Am J Sports Med 45)3:676-684 (Mar)

35. Wang CJ (2012) Extracorporeal shockwave therapy in musculoskeletal disorders. J Orthop Surg Res 20;7:11 (Mar)

36. Nepple JJ, Matava MJ et al (2009) Soft Tissue Injections in the Athlete. Sports Health 1(5):396-404 (Sep)

37. Reichl FX et al (2017) Comprehensive Analytics of Actovegin and Its Effect und Muscle Cells. Int J Sports Med 38:809-818

38. Sondergard SD et al (2016) Actovegin, a nonprohibited drug increases oxidative capacity in human skeletal muscle. Eur J Sport Sci 16(7):801-807

39. Steuer K, Steuer V (2017) Wie entsteht ein akutes Kompartmentsyndrom ohne Trauma? Trauma Berufskrankh 19:11. https://doi.org/10.1007/ s10039-017-0233-2

40. Subbu $R$ et al (2015) Timing of surgery for complete proximal hamstring avulsion injuries: successful clinical outcomes at 6 weeks, 6 months, and after 6 months of injury. Am J Sports Med 43(2):385-391. https://doi.org/10.1177/ 0363546514557938. Epub 2014 Nov 17

41. MadeAD, ReurinkG,GouttebargeVetal(2014)Outcome After Surgical Repair of Proximal Hamstring Avulsions: A Systematic Review. Am J Sports Med 43(11):2841-2851. 10.1177/0363546514555327. Epub2014 Nov 10 